\title{
Passage of Time in a Planck Scale Rooted Local Inertial Structure
}

\author{
Joy Christiar* \\ Wolfson College, Oxford University, Oxford OX2 6UD, United Kingdon
}

(Dated: 10 August 2003)

\begin{abstract}
It is argued that the 'problem of time' in quantum gravity necessitates a refinement of the local inertial structure of the world, demanding a replacement of the usual Minkowski line element by a $(4+2 n)$-dimensional pseudo-Euclidean line element, with the extra $2 n$ being the number of internal phase space dimensions of the observed system. In the refined structure, the inverse of the Planck time takes over the role of observer-independent conversion factor usually played by the speed of light, which now emerges as an invariant but derivative quantity. In the relativistic theory based on the refined structure, energies and momenta turn out to be invariantly bounded from above, and lengths and durations similarly bounded from below, by their respective Planck scale values. Along the external timelike world-lines, the theory naturally captures the 'flow of time' as a genuinely structural attribute of the world. The theory also predicts expected deviationssuppressed quadratically by the Planck energy - from the dispersion relations for free fields in the vacuum. The deviations from the special relativistic Doppler shifts predicted by the theory are also suppressed quadratically by the Planck energy. Nonetheless, in order to estimate the precision required to distinguish the theory from special relativity, an experiment with a binary pulsar emitting $\mathrm{TeV}$ range $\gamma$-rays is considered in the context of the predicted deviations from the second-order shifts.
\end{abstract}

PACS numbers: 04.50.+h, 03.30.+p, 04.60.-m

\section{INTRODUCTION}

Soon after the final formulation of general relativity, Einstein argued that quantum effects must modify the new theory of gravity [1]. Despite almost a century of debate, however, today there is little consensus on what eventual form such a modified theory should take [2]. One of the few notions that does enjoy unanimity in this context is the recurrent suggestion of a 'minimum length', which is usually taken to be the Planck length $l_{P}:=\sqrt{\hbar G_{N} / c^{3}}$. It is generally believed that the Planck scale (cf. p. 471 of [3]) marks a threshold beyond which the usual continuum description of spacetime is unlikely to survive. Already in the wake of Einstein's pioneering argument there existed a number of speculative proposals for discrete spacetimes. For instance, Heisenberg suggested that the spatial continuum should be replaced by a 'lattice world', composed of cells of a finite size [4, 5]. Later he abandoned the idea, however, concluding that the assumption of minimum length appeared to be incompatible with Lorentz invariance. Indeed, an elementary implication of Lorentz contraction reveals that an assumed minimum length in one inertial frame may have a different - and even vanishing - value in another.

It is now well known that in a remarkable paper Snyder 6] eventually resolved this apparent incompatibility between the existence of a minimum length and Lorentz invariance (see also [7]). In the modern parlance, Snyder's resolution amounts to finding a non-linear basis for the Poincaré algebra that allow two, instead of one, observerindependent scales; namely, the maximum speed $c$ as

\footnotetext{
*Electronic address: joy.christian@wolfson.oxford.ac.uk

${ }^{\dagger}$ Journal Reference: Int. J. Mod. Phys. D 13 (2004) 1037-1071
}

well as a minimum length $l_{P}$. Recent years have seen a revival of theories allowing two observer-independent scales based on such non-linear actions of the Poincaré group 8, 9, 10, 11]. Physically, these theories correspond to non-linear modifications of special relativity, allowing the low-energy photons in some cases to travel faster than the upper bound $c$. One may classify these neo-Snyder theories as 'bottom-up' attempts to construct quantum gravity, in a manner not too dissimilar from how general relativity was founded by Einstein on special relativity.

The theory developed in this paper shares the general 'bottom-up' philosophy of these approaches. However, it also fundamentally differs from them in a number of different ways. For instance, as expected of any framework underlying quantum gravity, the only fundamental scale in the theory proposed here is the Planck scale; although the vacuum speed of light remains an observerindependent absolute upper bound on speeds as before, it now plays only a secondary and derivative role. What is more, unlike the diversity of the two-scales theories, the theory developed here is unique, with energies and momenta invariantly bounded from above, and lengths and durations similarly bounded from below, by their respective Planck scale values. Away from the Planck scale the theory reduces in general, as any such theory must, to Einstein's special theory of relativity.

Perhaps most significantly, and at least compared to the two-scales theories (cf. [12, 13]), the theory proposed here is based on operationally better grounds, thereby retaining the original rationale of Einstein's one-scale theory. In particular, the central aim of the proposed theory is to eliminate the operationally questionable dualistic conception of time implicitly taken for granted in physical theories. This leads to a generalization of Lorentz invariance that is fundamentally different form either the conception of a 'broken Lorentz invariance'- 
as in some approaches to quantum gravity [14], which may give rise to a 'preferred frame'-or the conception of 'modified Lorentz invariance' - as in the two-scales theories [9], which may not give rise to a 'preferred frame'. By contrast, the generalized invariance employed in the present theory is - by its very essence-hostile to the notion of a 'preferred frame'. In fact, the employed invariance respects the principle of relativity fully across the conceptual and physical domains that are even broader than those in Einstein's theories of relativity.

As expected within at least some of the mainstream approaches to quantum gravity (for a review, see [15]), the theory proposed here also predicts (exact) deviationssuppressed quadratically by the Planck energy - from the dispersion relations for the propagation of free fields in the vacuum. In recent years it has become increasingly probable that theories predicting even such minute deviations from the dispersion relations may be subjected to observational tests (see [15, 16, 17, 18, 19] and references therein). In addition to these possibilities, we shall also attempt to estimate the precision required to distinguish the modified Doppler shifts predicted by the present theory from those predicted by special relativity, by considering an experiment with a binary pulsar emitting $\mathrm{TeV}$ range $\gamma$-rays.

One of the oldest issues in natural philosophy is 'the problem of change' [20]. Since the days of Aristotle, physics has been tremendously successful in explaining how the changes occur in the world, but largely oblivious to the deeper question of why do they occur at all. The situation has been aggravated by the advent of Einstein's theories of spacetime, since in these theories there is no room to accommodate a structural distinction between the past and the future [21] - a prerequisite for a true understanding of why the changes occur in the world. By contrast, the causal structure of the proposed theory below not only naturally distinguishes the future form the past, but also forbids inaction altogether, thereby providing an answer to the deeper question of change.

In what follows we begin with the conceptual basis of the proposed theory in Secs. II to V, culminating in a $(4+2 n)$-dimensional fundamental quadratic invariant, namely (30). The basic physical consequences of the theory erected on this new quadratic invariant are then spelt out in Sec. VI. In the following section, Sec. VII, we raise and answer the age-old question: 'How fast does time flow?' (see, e.g., 22]). Since the proposed theory naturally quantifies the motion of the present moment (or 'now') along timelike world-lines of observers, what we have is an experimentally verifiable complete theory of the local inertial structure that captures the 'flow of time' as a genuine attribute of the world. Finally, before concluding in Sec. IX, the options for possible experimental verifiability of the theory are explored in Sec.VIII.

\section{TIME IN SPECIAL RELATIVITY}

Recall that among Einstein's primary concerns while constructing special relativity were the notions of absolute time and relative motion. In particular, he noted that some of the assumptions underlying these concepts within the then existing framework of physics were operationally ill-founded. To rectify this inadequacy, he proposed an operationally better founded structure we now take to be the local inertial structure of the world. As is well known, this structure -later geometrically refined by Minkowski - is based on the following two postulates: (i) The laws governing the states of physical systems are insensitive to the state of motion of the reference coordinate system, as long as it remains inertial, and (ii) $N_{o}$ speed of a causally admissible influence can exceed the vacuum speed of light. The second of the two postulates can be succinctly restated as

$$
v \leq c
$$

with the understanding that it is a statement true for all local inertial observers regardless of their state of motion. This is reaffirmed by the law of composition of velocities proposed by Einstein: assuming all velocities involved have the same direction, the velocity of a material body, say $v^{k}(k=1,2$, or 3$)$, in one inertial frame is related to its velocity, say $v^{\prime k}$, in another frame, moving with a velocity $-v_{r}^{k}$ with respect to the first, by the relation

$$
v^{\prime k}=\frac{v^{k}+v_{r}^{k}}{1+c^{-2} v^{k} v_{r}^{k}} .
$$

Thus, as long as neither $v^{k}$ nor $v_{r}^{k}$ exceeds the causal upper bound $c, v^{\prime k}$ also remains within $c$. It is this absoluteness of $c$ that lends credence to the view that it is merely a conversion factor between the dimensions of time and space. This fact is captured most conspicuously by the four-dimensional quadratic invariant of spacetime,

$$
d t_{E}^{2}:=d t_{N}^{2}-c^{-2} d \mathbf{x}^{2},
$$

where $\mathbf{x}$ is a vector in the 3 -space, and $t_{N}$ and $t_{E}$ are the Newtonian (or absolute or coordinate) and Einsteinian (or proper or wristwatch) times, respectively. In natural units where $c$ is taken to be unity, this line element can be put in the familiar Minkowski form:

$$
d t_{E}^{2}:=d t_{N}^{2}-d \mathbf{x}^{2}=:-\eta_{a b} d x^{a} d x^{b} .
$$

The central tenet of special relativity is now simply the assertion that it is the time interval $d t_{E}$, and not the Newtonian interval $d t_{N}$, that is actually registered by an inertial observer between two nearby events in spacetime, with the causality restriction equivalent to (1) being

$$
d t_{E}^{2} \geq 0
$$

To date there exists no convincing experimental evidence contradicting the varied predictions of Einstein's theory. 
Despite the phenomenal empirical successes of special relativity, however, a closer inspection reveals that, as yet, Einstein's first postulate - the principle of relativity -is not fully respected within the current framework of physics. As we shall see, when thoroughly implemented, the principle necessitates generalizations of the upper bound (11) and the law of composition (2), which in turn demand a Planck scale amelioration of the metric (3).

\section{TIME IN QUANTUM GRAVITY}

To appreciate the above assertions, consider an object system, equipped with an ideal classical clock of unlimited accuracy, moving with a uniform velocity $\mathbf{v}$ in a Minkowski spacetime $\mathcal{M}$, from an event $\mathrm{e}_{1}$ at the origin of a reference frame to a nearby event $\mathrm{e}_{2}$ in the future light cone of $e_{1}$. For our purposes, it would suffice to refer to this system, say of $n$ degrees of freedom, simply as 'the clock' (cf. 23]). As it moves, the clock will also necessarily evolve by the virtue of its 'external' motion, say at a uniform rate $\boldsymbol{\omega}$, from one state, say $\mathrm{s}_{1}$, to another, say $\mathrm{s}_{2}$, within its own 'internal' relativistic phase space, say $\mathcal{N}$. This evolution of the clock - or rather that of its state - from $s_{1}$ to $s_{2}$ will, of course, trace out a unique trajectory in the phase space $\mathcal{N}$, which, as is customary for any state space, may be parameterized by the Newtonian time $t_{N}$ and viewed as a curve in the resulting $(1+2 n)$-dimensional extended phase space (or contact manifold) 24]. For simplicity, here we shall assume the phase space to be finite and $2 n$ dimensional; apart from possible mathematical encumbrances, however, nothing prevents the following reasoning to remain valid for the case of infinite dimensional phase spaces (e.g. for clocks made out of relativistic fields).

Now, one may think of the motion and evolution of the clock conjointly as taking place in a combined $(4+2 n)$ dimensional space, say $\mathcal{E}$, the elements of which may be called event-states and represented by pairs $\left(\mathrm{e}_{i}, \mathrm{~s}_{i}\right)$. Undoubtedly, it is this combined space that truly captures the complete specification of all possible physical attributes of the classical clock. Therefore, we may ask: What will be the time interval actually registered by the clock as it moves and evolves from the event-state $\left(\mathrm{e}_{1}, \mathrm{~s}_{1}\right)$ to the event-state $\left(\mathrm{e}_{2}, \mathrm{~s}_{2}\right)$ in this combined space $\mathcal{E}$ ? It is only by answering such a physical question that one can determine the correct topology and geometry of the combined space in the form of a metric analogous to (3).

Of course, the customary answer to the above question, in accordance with the spacetime metric (3), is simply

$$
\Delta t_{E}=\int_{e_{1}}^{e_{2}} \frac{1}{\gamma(v)} d t_{N}=\frac{\left|t_{2}-t_{1}\right|}{\gamma(v)}
$$

with the usual Lorentz factor

$$
1<\gamma(v):=\left(1-c^{-2} v^{2}\right)^{-\frac{1}{2}} .
$$

In other words, the customary answer is that the space $\mathcal{E}$ has a product topology, $\mathcal{E}=\mathcal{M} \times \mathcal{N}$, and-more to the point - the clock recording the duration $\Delta t_{E}$ in question is insensitive to the passage of time that marks the evolution of variables within its own phase space $\mathcal{N}$.

This may appear to be a perfectly adequate answer. After all, a classical clock is supposed to register the time external to itself, and the phase space of its evolution is an internal space, dependent on the physical attributes of the clock. In other words, the time measured by such a clock is presumed to be 'external', existing independently of it, whereas its dynamic evolution, although parameterizable by this background time, is viewed to be 'internal', specific to the clock itself. Such a dualistic conception of time has served us well in special relativistic physics. In fact, we encounter no serious difficulties with its implementation in physical theories - classical or quantaluntil quantum gravity is confronted head-on 25].

The formidable difficulties in constructing quantum gravity, of course, stem from the fact that the basic principles of general relativity and quantum mechanics are fundamentally at odds with each other [2]. One of the many ways these difficulties can manifest themselves is via the so-called 'problem of time' within the canonical approaches to the task [26]. The problem, essentially, is that, unlike in special and general theories of relativity, in classical and quantum mechanics time is treated as an external parameter, marking the evolution of a given physical system. Within the above approaches, most attempts that try to reconcile the dynamical role of time in relativity with the parametric role of time in mechanics seek to understand this external parameter in terms of some internal structure of the system itself [26].

Needless to say, to date no such attempt has succeeded in providing unequivocal understanding of the 'external' time that observers do register on their clocks. What is worse, the dichotomy of time into external versus internal time emphasized by most such approaches to quantum gravity only manages to obscure the real culprit lurking behind the difficulty; namely, the dichotomy of simultaneity into relative versus absolute simultaneity. Unlike in spacetime physics, where the unphysical notion of absolute simultaneity was eliminated by Einstein, in state-time physics, the notion remains axiomatic. It is in this sense, then, that Einstein's first basic postulatethe principle of relativity - is not as yet fully respected within the established framework of physics.

\section{PROPER TIME IN THE 'REST FRAME'}

Let us look at these assertions more closely by unpacking them once again with the help of the moving and evolving clock considered above. In the 'global inertial frame' [3] in which the clock is at rest, the proper time interval it is supposed to register is simply $d t_{E}=d t_{N}$. Using this Newtonian time $t_{N}$ as an external parameter, within this frame one can determine the phase space $\mathcal{N}$ for the dynamical evolution of the clock using the standard Hamiltonian prescription [27]. Of course, as 
long as we start with a Lorentz invariant action, the resulting phase space within each such frame - although not manifestly covariant - would be consistent with the principles of special relativity. Suppose now we consider time-dependent canonical transformations of the dimensionless phase space coordinates $\mathrm{y}^{\mu}\left(t_{N}\right)(\mu=1, \ldots, 2 n)$, expressed in Planck units, into coordinates $\mathrm{y}^{\prime \mu}\left(t_{N}\right)$ of the following general linear form:

$$
\mathrm{y}^{\prime \mu}\left(\mathrm{y}^{\mu}(0), t_{\mathrm{N}}\right)=\mathrm{y}^{\mu}(0)+\omega_{r}^{\mu}(\mathbf{y}(0)) t_{N}+b^{\mu},
$$

where $\omega_{r}^{\mu}$ and $b^{\mu}$ do not have explicit time dependence, and the reason for the subscript $r$ in $\omega_{r}^{\mu}$, which stands for 'relative', will become clear soon. Interpreted actively, these are simply the linearized solutions of the familiar Hamiltonian flow equations [24],

$$
\frac{d \mathrm{y}^{\mu}}{d t_{N}}=\omega_{r}^{\mu}\left(\mathbf{y}\left(t_{N}\right)\right):=\Omega^{\mu \nu} \frac{\partial H}{\partial \mathrm{y}^{\nu}}
$$

where $\boldsymbol{\omega}_{r}$ is the Hamiltonian vector field generating the flow, $\mathbf{y}\left(t_{N}\right)$ is a $2 n$-dimensional local Darboux vector in the phase space $\mathcal{N}, \boldsymbol{\Omega}$ is the symplectic 2 -form on $\mathcal{N}$, and $H$ is a Hamiltonian function governing the evolution of the clock. In the dual description, the vector field $\boldsymbol{\omega}_{r}$ generating the integral curves of (9) can be defined by

$$
\omega_{\mu}^{r} \mathbf{d y}^{\mu}:=\boldsymbol{\Omega}\left(\cdot, \boldsymbol{\omega}_{r}\right)=\mathbf{d} H \equiv \frac{\partial H}{\partial \mathrm{y}^{\mu}} \mathbf{d y}{ }^{\mu},
$$

with $\mathbf{d}$ being the exterior derivative. Since $\boldsymbol{\Omega}\left(\cdot, \boldsymbol{\omega}_{r}\right)$ with only one of the two slots of the 2 -form $\boldsymbol{\Omega}$ filled - is a 1-form dual to the vector field $\boldsymbol{\omega}_{r}$, its components are denoted by $\omega_{\mu}^{r}$. In other words, as in any phase space, the indices $\mu$ of the vector field $\omega_{r}^{\mu}$ is lowered, not by a possible metric on $\mathcal{N}$, but by the symplectic 2 -form $\Omega$. Being antisymmetric, however, the 2 -form $\boldsymbol{\Omega}$ is not suitable as a metric on $\mathcal{N}$. Fortunately, at least locally, one can define a Euclidean metric $\boldsymbol{\delta}$ on $\mathcal{N}$ to evaluate inner products. Using this metric, it is then easy to see from relations (9) and (10) that, when Hamiltonian happens to be the total energy of the system, the dimensionless magnitude of the vector field $\boldsymbol{\omega}_{r}$ is given by

$$
t_{P} \omega_{r}=\frac{d E}{E_{P}}
$$

where $E_{P}$ is the Planck energy, which, along with $t_{P}$, explicates the balance of dimensions on the two sides.

For a later use, let us make a few comments on the physical meaning of the Euclidean metric $\boldsymbol{\delta}$ on the phase space and the corresponding dimensionless line element

$$
d \mathbf{y}^{2}:=\delta_{\mu \nu} d \mathrm{y}^{\mu} d \mathrm{y}^{\nu} .
$$

In general, every symplectic manifold $(\mathcal{N}, \boldsymbol{\Omega})$ admits a family of compatible almost complex structures [29], but none of these need be integrable. When one is, it gives rise to a Riemannian metric on $(\mathcal{N}, \boldsymbol{\Omega})$ defined by $\mathbf{g}(\mathbf{X}, \mathbf{Y}):=\boldsymbol{\Omega}(\mathbf{X}, \mathbf{J Y})$, where $\mathbf{X}, \mathbf{Y}$ are two arbitrary vector fields and $\mathbf{J}$ is the complex structure. A prime example of such a complex manifold (known as a Kähler manifold) is the projective Hilbert space, with its well known Fubini-Study metric of a constant holomorphic curvature. Now, of course, this Fubini-Study metric gives rise to transition probabilities in quantum mechanics and reduces to the Euclidean metric $\boldsymbol{\delta}$ in the classical limit, whereas the corresponding symplectic form gives rise to the celebrated geometric phase factor and reduces to the classical symplectic form $\boldsymbol{\Omega}$ in the classical limit 30 . Therefore, the 'flat' Euclidean metric $\boldsymbol{\delta}$ (or the element (12)) of our phase space can be viewed as the 'quantum shadow' of the 'curved' quantum state space metric (i.e., of the Fubini-Study metric), also giving rise to transition probabilities - albeit of a rather trivial kind, yielding only extreme values of 0 or 1 (cf. 31]).

Now, since a phase space in general is simply a bare symplectic manifold, one may wonder about those clocks whose mathematical phase space descriptions do not require (or even admit) such a 'quantum shadow'. Since the 'non-metrical' phase space description of such a clock can only be recovered in a singular unphysical limit $(\hbar=0)$ from the metrical quantum state space description in terms of the Fubini-Study metric, in the present theory the pure symplectic description of a clock will be deemed 'too classical', and the corresponding clock will be deemed 'too crude' (i.e., not sensitive enough to resolve the Planck scale effects predicted by the present theory). Conversely, it has been argued by Klauder that any consistent quantization scheme inevitably ends up using the Euclidean metric, if only implicitly, and that in general it is impossible to ascribe any operational meaning to coordinatized phase space expressions for physical quantities, such as Hamiltonians, if the corresponding phase space does not admit the Euclidean metric [32, 33]. Therefore, in what follows it will be taken for granted that the phase space of our Planck scale sensitive clock is equipped with the 'quantum shadow metric' $\boldsymbol{\delta}$. In other words, in line with the 'bottom-up' philosophy advocated in the Introduction, it will be taken for granted that it is this remnant of quantum mechanics - this 'quantum shadow metric' $\boldsymbol{\delta}$ - that renders our clock sensitive to the Planck scale effects predicted by the present theory.

If we now denote by $\omega^{\mu}$ the uniform time rate of change of the canonical coordinates $\mathrm{y}^{\mu}$, then the time-dependent canonical transformations (8) imply the composition law

$$
\omega^{\prime \mu}=\omega^{\mu}+\omega_{r}^{\mu}
$$

for the evolution rates of the two sets of coordinates, with $-\omega_{r}^{\mu}$ interpreted as the rate of evolution of the transformed coordinates with respect to the original ones (in fact, Arnold 24], for example, calls $\boldsymbol{\omega}_{r}$ simply 'the phase velocity vector field'). Crucially for our purposes, what is implicit in the law (13) is the assumption that there is no upper bound on the rates of evolution of physical states. Indeed, successive transformations of the type (8) can be used, along with (13), to generate arbitrarily high 
rates of evolution for the state of the clock.

More pertinently, the assumed validity of the composition law (13) of evolution rates turns out to be equivalent to assuming absolute simultaneity within the $(1+2 n)$ dimensional extended phase space [24], say $\mathcal{O}$. To appreciate this fact, let us view $\mathcal{O}$ as an abstract manifold, and compare the causal structure of its elementswhich may be called occasions $o_{i}$, representing the pairs $o_{i}:=\left(t_{i}, s_{i}\right)$ - with the familiar causal structure of events in spacetime (see, e.g., [3]). It is clear that the causal relationships between two such occasions, say $o_{i}$ and $o_{j}$, fall into the following three mutually exclusive possibilities: (i) It is possible, in principle, for a state of a physical system to evolve from occasion $o_{j}$ to occasion $o_{i}$, in which case $o_{j}$ is said to be in the past of $o_{i}$. (ii) It is possible, similarly, for a state to evolve from occasion $o_{i}$ to occasion $o_{j}$, in which case $o_{j}$ is said to be in the future of $o_{i}$. (iii) It is impossible, in principle, for a state of a physical system to be at both occasions $o_{i}$ and $o_{j}$. Now, if we further assume the validity of the composition law (13), or equivalently the possibility of limitlessly high rates of evolution for physical states, then, in analogy with events in spacetime, the occasions in $\mathcal{O}$ belonging to the third category above would form a $2 n$-dimensional set (i.e. a phase space) defining the notion of absolute simultaneity with $o_{i}$. Thus in this case, within the $(1+2 n)$-dimensional manifold $\mathcal{O}$, the $2 n$-dimensional phase spaces simply constitute strata of 'hypersurfaces of simultaneity', much like the strata of spatial hypersurfaces within a Newtonian spacetime. Indeed, the extended phase spaces such as $\mathcal{O}$ are usually taken to be contact manifolds [24], with topology presumed to be a product of the form $\mathbb{R} \times \mathcal{N}$.

Thus, not surprisingly, the assumption of absolute time in contact spaces, in defiance of the relativity principle, is equivalent to the assumption of 'no upper bound' on the possible rates of evolution of physical states. Now, as a variant of the recurrent suggestion of 'a minimum length' (or of a 'minimum time'; cf. [28]) discussed in the Introduction, suppose we impose the following upper bound on the rates of evolution of the state of our clock:

$$
\left|\frac{d \mathbf{y}\left(t_{N}\right)}{d t_{N}}\right|=: \omega \leq t_{P}^{-1},
$$

where $t_{P}$ is the Planck time. Clearly, if this upper bound is to have any physical significance, it must hold for all possible evolving phase space coordinates $\mathrm{y}^{\mu}\left(t_{N}\right)$ discussed above. And that is amenable if and only if the composition law (13) is replaced by

$$
\omega^{\prime \mu}=\frac{\omega^{\mu}+\omega_{r}^{\mu}}{1+t_{P}^{2} \omega^{\mu} \omega_{r}^{\mu}}
$$

(no summation over $\mu$ - cf. the composition law (2)), which implies that as long as neither $\omega^{\mu}$ nor $\omega_{r}^{\mu}$ exceeds the causal upper bound $t_{P}^{-1}, \omega^{\prime \mu}$ also remains within $t_{P}^{-1}$.

Along with (15), if we now insist, as we must, on retaining the causal relationships among possible occasions in
$\mathcal{O}$ classified above, then, the usual positive definite product metric of the space $\mathcal{O}$ would have to be replaced by a pseudo-Euclidean indefinite metric defined by

$$
d t_{A}^{2}:=d t_{N}^{2}-t_{P}^{2} d \mathbf{y}^{2}
$$

or, in Planck units, by

$$
d t_{H}^{2}:=d t_{N}^{2}-d \mathbf{y}^{2}=:-\zeta_{\alpha \beta} d y^{\alpha} d y^{\beta}
$$

together with the causality condition

$$
d t_{A}^{2} \geq 0
$$

analogous to (5), where the line element $d \mathbf{y}^{2}$ is defined in (12), and, for a later use, we have renamed $d t_{A}$ in (17) by $d t_{H}$, with subscript $H$ standing for 'Hamiltonian' (or 'internal'). Thus, if the causal upper bound (14) is to be invariantly respected, then, even in the rest frame, the $a c$ tual proper duration registered by the clock would be $d t_{A}$, and not the usually supposed interval $d t_{N}$. Therefore, it is the time $t_{A}$, and not the Newtonian time $t_{N}$, that should appear in the Hamiltonian flow equations, such as (9), governing the evolution of states. In the resulting mechanics, different canonical coordinates that are evolving with nonzero relative rates would differ in general over which occasions are simultaneous with a given occasion. As unorthodox as this resulting picture may appear, it is an inevitable consequence of the upper bound (14).

Let us now return to the simple thought experiment considered in the previous section and ask: In the clock's rest frame, what will be the time interval registered by it as it evolves from occasion $o_{1}:=\left(t_{1}, s_{1}\right)$ to occasion $o_{2}:=\left(t_{2}, s_{2}\right)$ within the space $(\mathcal{O}, \zeta)$ ? According to the line element (16), the answer clearly is

$$
\Delta t_{A}=\int_{o_{1}}^{o_{2}} \frac{1}{\gamma(\omega)} d t_{N}=\frac{\left|t_{2}-t_{1}\right|}{\gamma(\omega)}
$$

were

$$
1<\gamma(\omega):=\left(1-t_{P}^{2} \omega^{2}\right)^{-\frac{1}{2}}
$$

Thus, if the state of the clock is at all evolving, we will have the phenomenon of 'time dilation' even in the rest frame. In particular, for a clock evolving at the rate $t_{P}^{-1}$, the time stands still! Similarly, we may now speak of a phenomenon of 'state contraction' in analogy with the familiar phenomenon of 'length contraction':

$$
\Delta \mathrm{y}^{\prime}=\omega \Delta t_{A}=\frac{\omega \Delta t_{N}}{\gamma(\omega)}=\frac{\Delta \mathrm{y}}{\gamma(\omega)} .
$$

It is worth emphasizing here that, as in ordinary special relativity, nothing is actually 'dilating' or 'contracting'. All that is being exhibited by these phenomena is that the two sets of mutually evolving canonical coordinates happen to differ over which occasions are simultaneous.

In addition to the light cones in spacetime bounded by the maximum possible speed $c$, we may now also speak 
of Planck cones in the $(1+2 n)$-dimensional space $(\mathcal{O}, \zeta)$ bounded by the maximum rate of evolution $t_{P}^{-1}$. Analogous to a light cone delimiting the allowed causal relations between a given event and other events in spacetime (cf. [3]), a Planck cone would delimit the causal relations between a given occasion $o_{i}$ and other occasions within the space $(\mathcal{O}, \zeta)$. Accordingly, the occasions that lie on the boundary of the 'future Planck cone' of $o_{i}$ defined by $t_{P}^{-1}$ can be reached by a physical state at $o_{i}$ if and only if it is evolving at the maximum possible rate $t_{P}^{-1}$. These occasions, and similarly defined occasions lying on the 'past Planck cone' of $o_{i}$, form a $2 n$-dimensional set within $(\mathcal{O}, \zeta)$, and may be said to be Planck-like related to $o_{i}$. Occasions that are on neither the past nor the future Planck cone of $o_{i}$ but lie interior to the Planck cones are also causally accessible by a physical state at $o_{i}$, and may be said to be time-like related to $o_{i}$. The remaining occasions lying exterior to the Planck cones comprise a causally forbidden $(1+2 n)$-dimensional set within $(\mathcal{O}, \zeta)$, and may be said to be state-like related to $o_{i}$.

\section{PROPER TIME IN A 'MOVING FRAME'}

So far we have used a specific Lorentz frame, namely the rest frame of the clock, to obtain expression (16) for the actual proper duration. For a frame with respect to which the same clock is uniformly moving, the expression for actual proper duration can be now obtained at once by using the Minkowski line element (3), yielding

$$
d t_{A}^{2}=d t_{N}^{2}-c^{-2} d \mathbf{x}^{2}-t_{P}^{2} d \mathbf{y}^{2} .
$$

If we now eliminate the explicit appearance of the speed of light $c$ by defining it as a Planck scale ratio $l_{P} / t_{P}$, then this actual proper duration can be rewritten as

$$
d t_{A}^{2}=d t_{N}^{2}-t_{P}^{2} d \mathbf{z}^{2},
$$

with the dimensionless $3+2 n$ vector $\mathbf{z}$ defined by

$$
\mathbf{z}:=l_{P}^{-1} \mathbf{x}+\mathbf{y} .
$$

The answer to the central question raised in Sec. III is now clear: The time interval actually registered by the clock as it moves and evolves from the event-state $\left(\mathrm{e}_{1}, \mathrm{~s}_{1}\right)$ to the event-state $\left(\mathrm{e}_{2}, \mathrm{~s}_{2}\right)$ in the combined space $\mathcal{E}$ is given, not by (6) as customarily assumed, but by

$$
\Delta t_{A}=\int_{\left(\mathrm{e}_{1}, \mathrm{~s}_{1}\right)}^{\left(\mathrm{e}_{2}, \mathrm{~s}_{2}\right)} \frac{1}{\gamma(\theta)} d t_{N}=\frac{\left|t_{2}-t_{1}\right|}{\gamma(\theta)},
$$

were, in accordance with the line element (23),

$$
1<\gamma(\theta):=\left(1-t_{P}^{2} \theta^{2}\right)^{-\frac{1}{2}},
$$

with

$$
\theta:=\left|\frac{d \mathbf{z}\left(t_{N}\right)}{d t_{N}}\right|=\left|l_{P}^{-1} \mathbf{v}+\boldsymbol{\omega}\right| .
$$

This answer for the actual proper duration registered by the clock between the event-states $\left(\mathrm{e}_{1}, \mathrm{~s}_{1}\right)$ and $\left(\mathrm{e}_{2}, \mathrm{~s}_{2}\right)$ comprises the central, empirically verifiable, claim of the theory proposed here. The fundamental reason behind such an overall 'time dilation' effect encoded in this answer is the fact that, according to the present theory, different inertial observers disagree in general, not just over which events occur simultaneously with a given event, but over which event-states occur simultaneously with a given event-state. Note, however, that away from the Planck scale, and/or for slow evolutions of states, the ameliorated gamma factor (26) duly reduces to the usual gamma factor (7) of special relativity, which of course presumes absolute simultaneity of physical states.

We are now in a position to isolate the two basic postulates on which the theory developed here can be erected in the manner analogous to the usual special relativity. In fact, the first of the two postulates can be taken to be Einstein's very own first postulate, as stated above, except that we must now revise what we mean by an inertial coordinate system. In normal practice, an inertial coordinate system is taken to be a system of four dimensions, one temporal and three spatial, moving uniformly in spacetime. In the present theory it is understood to be a system of $4+2 n$ dimensions, 'moving' uniformly in the combined space $\mathcal{E}$, with four being the external spacetime dimensions, and $2 n$ being the internal phase space dimensions of a system under scrutiny. Again, the internal dimensions of the object system can be either finite or infinite in number. The principle of relativity may now be restated as follows: $\left(\mathrm{i}^{\prime}\right)$ The laws governing the states of physical systems are insensitive to 'the state of motion', in the space $\mathcal{E}$, of the $(4+2 n)$-dimensional reference coordinate system, as long as it remains 'inertial'.

The second postulate on which the present theory is based is a generalization of the above stated second postulate of Einstein. According to it: (ii') No time rate of change of a dimensionless physical quantity expressed in Planck units can exceed the inverse of the Planck time. In particular, for the dimensionless quantity $\mathbf{z}$ defined above, this postulate may be succinctly restated as

$$
\theta \leq t_{P}^{-1},
$$

with understanding that this is a statement true for all local inertial observers regardless of their state of motion. That is to say, in order for this causal upper bound to be invariantly meaningful, the time rate of change $\theta^{I}$ of any such quantity $\mathrm{z}^{I}$ must satisfy the composition law

$$
\theta^{\prime I}=\frac{\theta^{I}+\theta_{r}^{I}}{1+t_{P}^{2} \theta^{I} \theta_{r}^{I}}
$$

analogous to (15), where $I=1,2, \ldots$, or $3+2 n$.

Note that away from the Planck scale and/or when $\boldsymbol{\omega}$ is negligible, $\boldsymbol{\theta} \approx l_{P}^{-1} \mathbf{v}$, and (28) duly reproduces (11). Moreover, for external directions, $I=1,2$, or 3 , (29) is identical to the composition law (2) for velocities, and for internal directions, $I=4,5, \ldots$, or $3+2 n$, it is identical to 
the similar composition law (15) for evolution rates. This means, in particular, that, despite the generalization, the constant ' $c$ ' still remains an observer-independent upper bound on admissible speeds. This invariance of ' $c$ ' will be reaffirmed later (cf. eq. (60) ) as a derivative notion.

With the composition law (29), it is now easy to see that, just as in Einstein's special relativity, the above two revised postulates inevitably lead to the actual proper duration (23), along with the causality condition (18). For the sake of conceptual clarity, so far in these expressions we have used the notations $t_{E}$ and $t_{N}$ for the proper and coordinate times, respectively. Employing more familiar notations for these notions of time and using Planck units, we now rewrite expression (23) as

$$
d \tau^{2}=d t^{2}-d \mathbf{z}^{2}=:-\xi_{A B} d z^{A} d z^{B},
$$

where the index $A=0, \ldots, 3+2 n$ runs along the $4+2 n$ dimensions of the pseudo-Euclidean manifold $(\mathcal{E}, \xi)$. This, then, according to the present theory, is the true quadratic invariant of the inertial structure of the world.

\section{REFINED LOCAL INERTIAL PHYSICS}

Having arrived at the above refined chart of the inertial structure, we shall now see how it is further justified by its amicable theoretical consequences. In particular, we shall see how, in the theory based on the refined structure, energies and momenta turn out to be invariantly bounded from above, and lengths and durations similarly bounded from below, by their respective Planck scale values.

\section{A. Permitted coordinate transformations}

The coordinate transformations in the combined space $(\mathcal{E}, \xi)$ analogous to the Lorentz transformations in the Minkowski spacetime that preserve the above fundamental quadratic invariant ([30) or (22) can be written as

$$
z^{A}=\Lambda_{B}^{A} z^{B}+b^{A}
$$

where $b^{A}$ and $\Lambda_{B}^{A}$ are constants, constrained by

$$
\Lambda_{C}^{A} \Lambda_{D}^{B} \xi_{A B}=\xi_{C D}
$$

At least for simple, finite dimensional, phase spaces, the coefficients $\Lambda_{B}^{A}$ can be easily determined. Consider, for example, a massive relativistic particle at rest (and hence also not evolving) with respect to a primed coordinate system in the external spacetime, which is moving with a uniform velocity $\mathbf{v}$ with respect to another unprimed coordinate system. Since, as it moves, the state of the particle will also be evolving in its six dimensional phase space (and since the evolution of a mechanical system is simply the continuous unfolding of canonical transformations [24]), we can view its motion and evolution together with respect to a $(4+6)$-dimensional unprimed coordinate system in the space $(\mathcal{E}, \xi)$, and thus view it to be moving and evolving, say, at an arbitrary combined rate $\boldsymbol{\theta}$ (cf. (27)). Now, from (31) we have the differential relations

$$
d z^{A}=\Lambda_{B}^{A} d z^{\prime B}
$$

Since $d \mathbf{z}^{\prime}$ vanishes in the present case, these reduce to

$$
d \mathrm{z}^{I}=\Lambda_{0}^{I} d t^{\prime} \quad(I=1,2, \ldots, 9=3+6),
$$

and $\quad d t=\Lambda_{0}^{0} d t^{\prime}$.

Now, dividing (34) by (35) we have one relation between the coefficients $\Lambda^{I}{ }_{0}$ and $\Lambda_{0}^{0}$,

$$
\Lambda_{0}^{I}=\theta^{I} \Lambda_{0}^{0}
$$

and setting $C=D=0$ in (32) we have another,

$$
-1=\Lambda_{0}^{A} \Lambda_{0}^{B} \xi_{A B}=-\left(\Lambda_{0}^{0}\right)^{2}+\sum_{I=1}^{3+6}\left(\Lambda_{0}^{I}\right)^{2} .
$$

The solution of these two simultaneous equations is

$$
\Lambda_{0}^{0}=\gamma(\theta) \text { and } \Lambda_{0}^{I}=\gamma(\theta) \theta^{I},
$$

with $\gamma(\theta)$ given by (26). As in the familiar case of Lorentz transformations, hear also the other $\Lambda_{B}^{A}$ can be determined uniquely only up to arbitrary rotations. One convenient choice that would satisfy the constraint (32) is

$$
\Lambda_{J}^{0}=\gamma(\theta) \theta_{J} \quad \text { and } \quad \Lambda_{J}^{I}=\delta_{J}^{I}+\frac{\theta^{I} \theta_{J}}{\boldsymbol{\theta}^{2}}[\gamma(\theta)-1]
$$

\section{B. Bounds on lengths and durations}

Let us now restrict to the following two very special cases of only external spatio-temporal transformations:

$$
\begin{aligned}
d \mathrm{x}^{k} & =\gamma(v, \omega) d \mathrm{x}^{\prime k} \quad(k=1,2, \text { or } 3), \\
\text { and } \quad d t & =\gamma(v, \omega) d t^{\prime},
\end{aligned}
$$

where, reactivating the units for clarity, we have written

$$
\gamma(\theta) \equiv \gamma(v, \omega)=\left(1-c^{-2} v^{2}-t_{P}^{2} \omega^{2}\right)^{-\frac{1}{2}}
$$

Using (11), this gamma factor can also be written as

$$
\gamma(v, \omega) \equiv \gamma(v, d E)=\left[1-\frac{v^{2}}{c^{2}}-\frac{(d E)^{2}}{E_{P}^{2}}\right]^{-\frac{1}{2}}
$$

If we now assume that the difference of particle energy between the two coordinate systems is small but finite, that is, if we assume that

$$
\frac{d E}{E_{P}} \sim \frac{\Delta E}{E_{P}}=\frac{E^{\prime}-E}{E_{P}}
$$


then the gamma factor (43) takes the useful form

$$
\gamma(v, \omega) \sim \gamma(v, \Delta E)=\left[1-\frac{v^{2}}{c^{2}}-\frac{\left(E^{\prime}-E\right)^{2}}{E_{P}^{2}}\right]^{-\frac{1}{2}} .
$$

Now (40) and (41) are clearly the infinitesimal counterparts of the refined 'length contraction' and 'time dilation' relations. For a small but finite distance in the direction of the relative velocity $\mathbf{v}$, the first of these two relations can be rewritten as

$$
\Delta \mathrm{x}^{\prime}=\Delta \mathrm{x} \sqrt{1-\frac{v^{2}}{c^{2}}-l_{P}^{2}\left(\frac{\Delta \mathrm{x}-\Delta \mathrm{x}^{\prime}}{\Delta \mathrm{x}^{\prime} \Delta \mathrm{x}}\right)^{2}} .
$$

Here we have dropped the index on $\Delta \mathrm{x}$ and used

$$
\frac{E^{\prime}-E}{E_{P}}=\frac{l_{P}}{\Delta \mathrm{x}^{\prime}}-\frac{l_{P}}{\Delta \mathrm{x}},
$$

which follows from an inversion of units analogous to the one routinely used in high energy physics. The 'length contraction' expression (46) can be exactly solved for the 'contracted' length $\Delta \mathrm{x}^{\prime}$ in terms of the 'uncontracted' length $\Delta \mathrm{x}$. However, the solutions of (46) provided by the computer software Maple are far too long and complicated to be reproduced here. Fortunately, exact solutions of (46) are not necessary for our purposes of demonstrating the existence of a lower bound on lengths.

We begin with the assumption that the 'contracted' length $\Delta \mathrm{x}^{\prime}$ is negligibly smaller than the 'uncontracted' length $\Delta \mathrm{x}$. The question then is, how small the 'contracted' length can get? In other words, is there an absolute lower bound on lengths? The answer, according to the present theory, is, yes, there is a lower bound, and it is no other than $l_{P}$. To see this, note that under the assumption $\Delta \mathrm{x}^{\prime} \ll \Delta \mathrm{x}$, the relation (46) reduces to

$$
\Delta \mathrm{x}^{\prime}=\Delta \mathrm{x} \sqrt{1-\frac{v^{2}}{c^{2}}-\frac{l_{P}^{2}}{\left(\Delta \mathrm{x}^{\prime}\right)^{2}}} .
$$

Squaring both sides of this equation yields

$$
\left(\Delta \mathrm{x}^{\prime}\right)^{4}-\left(1-\frac{v^{2}}{c^{2}}\right)(\Delta \mathrm{x})^{2}\left(\Delta \mathrm{x}^{\prime}\right)^{2}+(\Delta \mathrm{x})^{2} l_{P}^{2}=0 .
$$

The only positive real root of this quartic equation for $\Delta \mathrm{x}^{\prime}$ that gives the correct physical limit

$$
\lim _{\Delta \mathrm{x} \gg l_{P}} \Delta \mathrm{x}^{\prime}=\Delta \mathrm{x} \sqrt{1-\frac{v^{2}}{c^{2}}}=\frac{\Delta \mathrm{x}}{\gamma(v)}
$$

is the following expression for 'length contraction',

$$
\Delta \mathrm{x}^{\prime}=\Delta \mathrm{x} \sqrt{\frac{1}{2}\left(1-\frac{v^{2}}{c^{2}}\right)+\sqrt{\frac{1}{4}\left(1-\frac{v^{2}}{c^{2}}\right)^{2}-\frac{l_{P}^{2}}{(\Delta \mathrm{x})^{2}}}}
$$

provided the reality condition

$$
\frac{1}{4}\left(1-\frac{v^{2}}{c^{2}}\right)^{2} \geq \frac{l_{P}^{2}}{(\Delta \mathrm{x})^{2}}
$$

is satisfied. Substituting this last inequality back into the solution (51) then gives

$$
\Delta \mathrm{x}^{\prime} \geq \sqrt{l_{P} \Delta \mathrm{x}} .
$$

Thus, as long as $\Delta \mathrm{x}$ is chosen to be greater than $l_{P}$, the 'contracted' length $\Delta \mathrm{x}^{\prime}$ also remains greater than $l_{P}$. That is to say, along with the upper bound implied by the condition $\gamma(v, \omega)>1$, the 'contracted' length remains invariantly bounded from below as well as from above:

$$
\Delta \mathrm{x}>\Delta \mathrm{x}^{\prime}>l_{P} .
$$

Starting again from the infinitesimal expression 41) now for 'time dilation' - and using almost identical line of arguments as the case above (along with the assumption $\Delta \tau \ll \Delta t$ for the 'dilated' time), we arrive, in analogous manner, at a refined expression for 'time dilation',

$$
\Delta \tau=\Delta t \sqrt{\frac{1}{2}\left(1-\frac{v^{2}}{c^{2}}\right)+\sqrt{\frac{1}{4}\left(1-\frac{v^{2}}{c^{2}}\right)^{2}-\frac{t_{P}^{2}}{(\Delta t)^{2}}}},
$$

together with the corresponding invariant bounds on the 'dilated' or proper time $\Delta \tau$ :

$$
\Delta t>\Delta \tau>t_{P} .
$$

\section{Reaffirming the bound on velocities}

So far we have not assumed or proved explicitly that the constant ' $c$ ' is an upper bound on possible speeds. As emphasised before (cf. comments after (29)), in the present theory the upper bound ' $c$ ' and its observerindependence turn out to be derivative notions. To see this explicitly, consider the ratio of 'contracted' length (51) and 'dilated' duration (55); that is, consider

$$
u^{\prime}=u \sqrt{\frac{\frac{1}{2}\left(1-\frac{v^{2}}{c^{2}}\right)+\sqrt{\frac{1}{4}\left(1-\frac{v^{2}}{c^{2}}\right)^{2}-\frac{l_{P}^{2}}{(\Delta \mathrm{x})^{2}}}}{\frac{1}{2}\left(1-\frac{v^{2}}{c^{2}}\right)+\sqrt{\frac{1}{4}\left(1-\frac{v^{2}}{c^{2}}\right)^{2}-\frac{t_{P}^{2}}{(\Delta t)^{2}}}}},
$$

where we have defined 'velocities' $u$ and $u^{\prime}$ as

$$
u:=\frac{\Delta \mathrm{x}}{\Delta t} \quad \text { and } \quad u^{\prime}:=\frac{\Delta \mathrm{x}^{\prime}}{\Delta \tau} .
$$

It is clear from these definitions that, for an arbitrary $\Delta \mathrm{x}^{\prime}$ subject to the bounds (54), $u^{\prime}$ would be the largest possible velocity when $\Delta \tau$ happens to be the smallest possible duration, which, in turn, for arbitrary but finite $v$ and $\Delta t$ (according to (55)), is given by the condition

$$
\frac{1}{4}\left(1-\frac{v^{2}}{c^{2}}\right)^{2}=\frac{t_{P}^{2}}{(\Delta t)^{2}} .
$$


Consequently, substituting this condition back into the ratio (57) gives the following upper bound on $u^{\prime}$ :

$$
u^{\prime} \leq u \sqrt{1+\sqrt{1-c^{2} u^{-2}}} .
$$

Thus, as long as $u$ does not exceed $c$ (and the resulting upper bound remains real), $u^{\prime}$ also remains within $c$. In other words, in the present theory $c$ retains its usual status of the observer-independent upper bound on causally admissible speeds, albeit in a rather derivative manner.

\section{Elements of particle mechanics}

Let us now take the mass of the particle considered above to be $m$. Then, just as in the standard special relativity 34], the fundamental quadratic invariant (30) leads to the 'momentum space' quadratic invariant

$$
\xi_{A B} \mathcal{P}^{A} \mathcal{P}^{B}=-m^{2} c^{2},
$$

provided we define the $4+2 n$ momentum $\mathcal{P}$ as

$$
m \frac{d z^{A}}{d \tau}=: \mathcal{P}^{A}:=\left(E / c, P^{I}\right),
$$

where the energy of the particle is now defined as

$$
E:=\gamma(v, d E) m c^{2},
$$

with $\gamma(v, d E)$ given by (43), and the $3+2 n$ momentum $P^{I}(I=1,2, \ldots, 3+2 n)$ of the particle is defined as

$$
\mathbf{P}:=\mathbf{P}_{\text {ext }}+\mathbf{P}_{\text {int }},
$$

with

$$
\mathbf{P}_{e x t} \equiv \mathbf{p}:=\gamma(v, d E) m \mathbf{v}
$$

and

$$
\mathbf{P}_{\text {int }}:=\gamma(v, d E) m l_{P} \boldsymbol{\omega} .
$$

Clearly, apart from the modified gamma factor, the expression (63) of energy is no different from the familiar one. The expression (64) for momentum, on the other hand, has two parts: an 'external' part $\mathbf{P}_{\text {ext }}$ and an 'internal' part $\mathbf{P}_{\text {int }}$. Again, apart from the modified gamma factor, the external momentum $\mathbf{P}_{e x t}$ is no different from the familiar one, and hence in what follows it will be denoted simply by $\mathbf{p}$. On the other hand, the notion of the internal $2 n$ momentum $\mathbf{P}_{\text {int }}$ is novel, and its physical meaning is as follows: Recall that $\boldsymbol{\omega}$ in (66) is simply the Hamiltonian vector field, which Arnold [24] occasionally refers to as 'the phase velocity vector field'. In the similar manner, $\mathbf{P}_{\text {int }}$ may be referred to as the phase momentum vector field on $\mathcal{N}$. Its usefulness will become clear soon.

Substituting now the explicit expression for $\mathcal{P}$ from (62) into the quadratic invariant (61), we obtain the following modified expression for the dispersion relation:

$$
|\mathbf{p}|^{2} c^{2}+m^{2} c^{4}=E^{2}-\left|\mathbf{P}_{i n t}\right|^{2} c^{2} .
$$

Using the definitions (66) for $\mathbf{P}_{\text {int }}$ and (63) for energy, along with the equations (10) and (11) for dynamics, the 1-form associated with the vector field $\mathbf{P}_{\text {int }}$ (which we denote also by $\mathbf{P}_{\text {int }}$ for convenience) can be written as

$$
\mathbf{P}_{\text {int }}=\frac{E \mathbf{d} E}{c E_{P}} .
$$

Substituting this into (67), we finally arrive at the refined dispersion relation between energies and momenta:

$$
p^{2} c^{2}+m^{2} c^{4}=E^{2}\left[1-\frac{(d E)^{2}}{E_{P}^{2}}\right] .
$$

It is worth noting that this is an exact relation between energies and momenta. We shall return to it in Sec. VIII to discuss how it can be experimentally verified. As a consistency check, note also that in the rest frame of the massive particle both $\mathbf{p}$ and $\mathbf{d} E$ vanish identically, and (69) reproduces the famous mass-energy equivalence:

$$
E=m c^{2} .
$$

If we now define refined energy $E_{R}$ as

$$
E_{R}:=E \sqrt{1-\frac{(d E)^{2}}{E_{P}^{2}}},
$$

then (69) takes the following perspicuous form:

$$
E_{R}^{2}=p^{2} c^{2}+m^{2} c^{4} .
$$

Since $E_{R} \rightarrow E \rightarrow m c^{2}$ as $p \rightarrow 0$, in the present theory this relation plays the role similar to that played by the usual dispersion relation in special relativity.

\section{E. Covariant conservation laws}

Consider an isolated system of mass $m_{\text {sys }}$ composed of a number of constituents undergoing an internal reaction. In view of the quadratic invariant (61), and considering what we have learned from special relativity 34], it is only natural to assume that the $4+2 n$ momentum $\mathcal{P}_{\text {sys }}$ of the system would be conserved in such a reaction,

$$
\Delta \mathcal{P}_{\text {sys }}=0
$$

where $\Delta$ denotes the difference between the initial and final states of the reaction, and $\mathcal{P}_{\text {sys }}$ is defined by

$$
m_{\text {sys }} \frac{d z^{A}}{d \tau}=: \mathcal{P}_{\text {sys }}^{A}:=\left(E_{\text {sys }} / c, p_{\text {sys }}^{k}, P_{\text {sys }}^{\mu}\right),
$$

with $k=1,2,3$ being the external three dimensions and $\mu=4,5, \ldots, 3+2 n$ being the phase space dimensions of the system as a whole. It is clear from this definition that, since $d z^{A}$ is a $(4+2 n)$-vector whereas $m_{\text {sys }}$ and $d \tau$ 
are invariants, $\mathcal{P}_{\text {sys }}^{A}$ is also a $(4+2 n)$-vector, and hence transforms under (33) as

$$
\mathcal{P}_{\text {sys }}^{\prime A}=\Lambda_{B}^{A} \mathcal{P}_{\text {sys }}^{B} .
$$

Moreover, since $\Lambda$ does not depend on anything but the permitted coordinate transformation being performed in the space $(\mathcal{E}, \xi)$, the difference on the left hand side of (73) is also a $(4+2 n)$-vector, and therefore transforms as

$$
\Delta \mathcal{P}_{\text {sys }}^{\prime A}=\Lambda_{B}^{A} \Delta \mathcal{P}_{\text {sys }}^{B} .
$$

Thus, if the conservation law (73) holds for one set of $(4+2 n)$-dimensional coordinates in $(\mathcal{E}, \xi)$, then, according to (76), it does so for all $(4+2 n)$-dimensional coordinates related by the transformations (31). Care must be taken, however, when implementing such a deceptively simple conservation law in a real physical reaction.

To appreciate one of the main subtleties, let us unpack (73) into its external, internal, and constituent parts:

$$
0=\Delta \mathcal{P}_{\text {sys }}=\left(\Delta E_{\text {sys }} / c, \Delta \mathbf{p}_{\text {sys }}, \Delta \mathbf{P}_{\text {int }}^{\text {sys }}\right),
$$

with

$$
\Delta E_{\text {sys }}:=\sum_{f} E_{f}-\sum_{i} E_{i}
$$

and

$$
\Delta \mathbf{p}_{s y s}:=\sum_{f} \mathbf{p}_{f}-\sum_{i} \mathbf{p}_{i},
$$

where the indices $f$ and $i$ stand for the final and initial number of constituents of the system. Thus, as in the special theory of relativity (and unlike in the two-scales theories 35|), in the present theory energies and momenta remain additive. What is crucial to note, however, is that $\mathbf{P}_{\text {int }}^{\text {sys }}$, being the phase momentum vector field of the system as a whole, does not, in general, warrant decomposition analogous to (79) into constituent parts.

Another aspect the conservation law (73) worth noting is that all of its $4+2 n$ components stand or fall together: any one of them entails all the others. Since $\Delta \mathcal{P}_{\text {sys }}$ is a $(4+2 n)$-vector, the relativity principle in the form of the coordinate transformations (76) dictates that, if any one of the components of the $(4+2 n)$-momentum $\mathcal{P}_{\text {sys }}$ is invariantly conserved in a reaction, then the entire vector, with all of its components, must also be so conserved. In particular, if the energy $E_{\text {sys }}$ is conserved in a reaction, then so is the momentum $\mathbf{p}_{\text {sys }}$, and vise versa.

Sometimes it is asserted [17] that the desire to maintain unmodified energy-momentum conservation laws accompanying a modified dispersion relation such as (69) may come at a price of introducing a preferred class of inertial observers in a theory; and, conversely, avoiding such a preferred class of observers would necessarily lead to a modification of conservation laws. However, it is clear form the above discussion that, in the present theory, not only are there no preferred class of observers, but also the conservation laws of special relativity - at least in the external sector-remain essentially unchanged.

\section{F. Bounds on energies and momenta}

Not surprisingly, analogous to the lower bounds on lengths and durations, in the present theory energies and momenta are also invariantly bounded from above. In the case of energy, this can be seen from the definition of energy (63) itself. Using (45), and an assumption similar to the one we used for the 'contracted' length (again employed for the same practical reasons), namely $E^{\prime} \gg E$, the definition (63) of energy can be expressed as

$$
E^{\prime}=\frac{E}{\sqrt{1-\frac{v^{2}}{c^{2}}-\frac{\left(E^{\prime}\right)^{2}}{E_{P}^{2}}}} .
$$

Squaring both sides of this equation leads to the following quartic equation for energy:

$$
E^{\prime 4}-\left(1-\frac{v^{2}}{c^{2}}\right) E^{\prime 2} E_{P}^{2}+E^{2} E_{P}^{2}=0 .
$$

The only positive real root of this equation for $E^{\prime}$ that gives the correct physical limit

$$
\lim _{E \ll E_{P}} E^{\prime}=E\left(1-c^{-2} v^{2}\right)^{-\frac{1}{2}}=\gamma(v) E
$$

is the following refined expression for energy:

$$
E^{\prime}=E \sqrt{\left(1-\frac{v^{2}}{c^{2}}\right) \frac{E_{P}^{2}}{2 E^{2}}-\sqrt{\left(1-\frac{v^{2}}{c^{2}}\right)^{2} \frac{E_{P}^{4}}{4 E^{4}}-\frac{E_{P}^{2}}{E^{2}}}}
$$

provided the reality condition

$$
\left(1-\frac{v^{2}}{c^{2}}\right)^{2} \frac{E_{P}^{4}}{4 E^{4}} \geq \frac{E_{P}^{2}}{E^{2}}
$$

is satisfied. Substituting this last inequality back into the solution (83) then gives

$$
E^{\prime} \leq \sqrt{E_{P} E} .
$$

Thus, as long as $E$ does not exceed $E_{P}, E^{\prime}$ also remains within $E_{P}$. That is, along with the lower bound implied by the condition $\gamma(v, \Delta E)>1$, the energy remains invariantly bounded from both below and above:

$$
E<E^{\prime}<E_{P} .
$$

Note that, unlike the evaluations of limit (50), the closed form evaluation of the limit (82) is not straightforward and requires an iterative use of the L'Hôpital's rule. In other words, the dependence of $E^{\prime}$ on the vanishing ratio $E / E_{P}$ is rather subtle compared to, say, the dependence of $\Delta \mathrm{x}^{\prime}$ on the analogous ratio $l_{P} / \Delta \mathrm{x}$.

Starting again from the definition (65) for momentum, and using almost identical line of arguments as the case above, we can arrive at an analogously refined expression 
for momentum as follows. As in special relativity, the ratio of (65) and (63) gives the identity

$$
\frac{p c}{E}=\frac{v}{c},
$$

which, upon using the Planck momentum $k_{P}:=E_{P} / c$, can also be written as

$$
\frac{E}{E_{P}}=\frac{p c}{k_{P} v}
$$

Using (45) and this identity into (65) (along with the assumption $p^{\prime} \gg p$ ) and then squaring both sides of (65) leads to the following quartic equation for momentum:

$$
p^{4}-\left(1-\frac{v^{2}}{c^{2}}\right) \frac{v^{2}}{c^{2}} k_{P}^{2} p^{2}+\frac{v^{2}}{c^{2}} k_{P}^{2} p^{2}=0 .
$$

The only positive real root of this equation that gives the correct physical limit

$$
\lim _{p v \ll k_{P} c} p^{\prime}=p\left(1-c^{-2} v^{2}\right)^{-\frac{1}{2}}=\gamma(v) p
$$

is the following refined expression for momentum:

$p^{\prime}=p \sqrt{\left(1-\frac{v^{2}}{c^{2}}\right) \frac{k_{P}^{2}}{2 p^{2}} \frac{v^{2}}{c^{2}}-\sqrt{\left(1-\frac{v^{2}}{c^{2}}\right)^{2} \frac{k_{P}^{4}}{4 p^{4}} \frac{v^{4}}{c^{4}}-\frac{k_{P}^{2}}{p^{2}} \frac{v^{2}}{c^{2}}},}$

provided the reality condition

$$
\left(1-\frac{v^{2}}{c^{2}}\right)^{2} \frac{k_{P}^{4}}{4 p^{4}} \frac{v^{4}}{c^{4}} \geq \frac{k_{P}^{2}}{p^{2}} \frac{v^{2}}{c^{2}}
$$

is satisfied. Substituting this last inequality back into the solution (91) then gives

$$
p^{\prime} \leq \sqrt{k_{P} p} \times \sqrt{\frac{v}{c}} .
$$

Thus, as long as $v$ does not exceed $c$ and $p$ does not exceed $k_{P}, p^{\prime}$ also remains within $k_{P}$. That is, along with the lower bound implied by the condition $\gamma(v, \Delta E)>1$, the momentum remains invariantly bounded from above as well as from below:

$$
p<p^{\prime}<k_{P} \text {. }
$$

In summary, we conclude from the bounds (54), (156), (86), and (94) that, in the present theory, all physical quantities of interest are invariantly bounded by their respective Planck scale counterparts.

\section{G. Massless particles and Doppler shifts}

In Einstein's special relativity, Doppler shifts provide one of the most transparent demonstrations of how the principle of relativity holds in nature. Despite the highly nonlinear character of the 'external' relations hidden in the overall linear transformations (31), the same remains true here, albeit with Planck scale enhanced Doppler shifts. In order to appreciate this, let us first spell out the general properties of massless particles in the theory.

It is evident from the invariant (61) that energy and momentum of any such massless particle must satisfy

$$
E^{2}=|\mathbf{P}|^{2} c^{2} \equiv|\mathbf{p}|^{2} c^{2}+\left|\mathbf{P}_{\text {int }}\right|^{2} c^{2},
$$

which - as we saw in the case of the general dispersion relation 69 - is equivalent to the relation

$$
p^{2} c^{2}=E^{2}\left[1-\frac{(d E)^{2}}{E_{P}^{2}}\right] \text {. }
$$

Using the identity (87), it is then easy to see that the massless particle must move with the speed

$$
v=c \sqrt{1-\frac{(d E)^{2}}{E_{P}^{2}}}
$$

Thus, any massless particle such as a photon with nonzero energy always moves with a speed somewhat less than $c$, and if the difference $d E$ in energy happens to approach the Planck energy $E_{P}$, then it hardly moves.

Consider now a receiver receding from a photon source with a uniform velocity v. As a given photon of energy $E$ propagates, it would also evolve in its phase space $\mathcal{N}$ with a uniform rate, say $\boldsymbol{\omega}$, relative to the canonical coordinates chosen in $\mathcal{N}$ as a part of the reference frame for the source (recall that, in the present theory, a frame of reference is a $(4+2 n)$-dimensional object). If we now take the combined relative 'velocity' between the source and the receiver to be $\boldsymbol{\theta}$ as defined by (27), then the energy of the photon observed by the receiver would be simply

$$
E^{\prime}=\gamma(\theta)\left[E-l_{P} \boldsymbol{\theta} \cdot \mathbf{P}\right]
$$

which follows from a transformation analogous to (31) applied to the photon's $4+2$ n momentum $\mathcal{P}$ defined by (62). If we next assume that the angle between the $(4+2 n)$-dimensional vectors $\boldsymbol{\theta}$ and $\mathbf{P}$ is $\Phi$, then, as a result of the relation (95), this transformation simplifies to

$$
E^{\prime}=\gamma(\theta) E\left[1-t_{P} \theta \cos \Phi\right]
$$

Of course, an external experimenter cannot be expected to have a direct access to either $\theta$ or $\Phi$, but, as we shall see, the knowledge of either $\theta$ or $\Phi$ is not necessary.

Now, to see how the principle of relativity works in the present theory, let us ask what would be the energy of the photon observed by the receiver if we view the source to be receding rather than the receiver. In other words, we now analyze the problem using the $(4+2 n)$ dimensional reference frame of the receiver rather than 
that of the source. The answer, not surprisingly, is given by the inverse transformation

$$
E=\gamma(\theta)\left[E^{\prime}+l_{P} \boldsymbol{\theta} \cdot \mathbf{P}^{\prime}\right],
$$

which, similarly to (99), reduces to

$$
E=\gamma(\theta) E^{\prime}\left[1+t_{P} \theta \cos \Phi^{\prime}\right],
$$

where $\Phi^{\prime}$ is the angle between the $(4+2 n)$-dimensional vectors $\boldsymbol{\theta}$ and $\mathbf{P}^{\prime}$. If we now make the substitutions $\theta^{\prime I}=-t_{P}^{-1} \cos \Phi^{\prime}, \theta^{I}=-t_{P}^{-1} \cos \Phi$, and $\theta_{r}^{I}=\theta$ into the composition law (29), then we expectedly arrive at the aberration relation between the angles $\Phi^{\prime}$ and $\Phi$ :

$$
\cos \Phi^{\prime}=\frac{\cos \Phi-t_{P} \theta}{1-t_{P} \theta \cos \Phi} .
$$

Upon using this relation, along with a use of (26), it is then easy to see that (99) and (101) are, in fact, identical relations between the observed and source energies of the photon. Thus, as in special relativity, the principle of relativity here as well renders it impossible to distinguish between the motions of the source or the receiver.

As promised, let us now obtain the relation between the overall angle $\Phi$ and the external angle, say $\phi$, which is the angle between the relative velocity $\mathbf{v}$ and photon momentum p. This relation between $\Phi$ and $\phi$ can be obtained by expanding the dot product $t_{P} \boldsymbol{\theta} \cdot \mathbf{P}$ as

$$
t_{P} \theta \cos \Phi=\frac{1}{|\mathbf{P}|}\left(c^{-1} v p \cos \phi+t_{P} \omega\left|\mathbf{P}_{i n t}\right|\right),
$$

where we have used the fact that - as is evident from the definition (66) $-\boldsymbol{\omega}$ and $\mathbf{P}_{\text {int }}$ are parallel vectors. Using (95), (96), (63), (66), (68), (11), and (44), this expansion can be reduced to

$$
t_{P} \theta \cos \Phi=\frac{\left(E^{\prime}-E\right)^{2}}{E_{P}^{2}}+\left(\frac{v}{c} \cos \phi\right) \sqrt{1-\frac{\left(E^{\prime}-E\right)^{2}}{E_{P}^{2}}} .
$$

Substituting this relation between the angles $\Phi$ and $\phi$ back into (99) and using (45) then gives the desired ratio

$$
\frac{E^{\prime}}{E}=\frac{\varepsilon^{\prime}\left[\varepsilon^{\prime}-\frac{v}{c} \cos \phi\right]}{\sqrt{\left(\varepsilon^{\prime}\right)^{2}-\frac{v^{2}}{c^{2}}}}
$$

where we have defined

$$
\varepsilon^{\prime}:=\sqrt{1-\frac{E^{2}}{E_{P}^{2}}\left(1-\frac{E^{\prime}}{E}\right)^{2}},
$$

which clearly becomes unity for $E \ll E_{P}$, thus reducing (105) to the familiar expression for Doppler shifts. In fact, it can be shown that the formula (105) rigorously reduces to the familiar expression in the $E / E_{P} \rightarrow 0$ limit.

Even without solving the relation (105) for $E^{\prime}$ in terms of $E$, since $\varepsilon^{\prime}<1$, one may be tempted to infer that at sufficiently high energies any red-shifted photons are somewhat more red-shifted according to (105) than predicted by special relativity. This, however, would be a mistake. It is clear that no such definite statement can be made for the case of receiver approaching toward rather than receding from the source -i.e., for the blueshifted photons - without actually solving the relation (105). Unfortunately, (105) is hopelessly nonlinear to be solved easily. In fact, its solution is a root of an eighth order polynomial equation in the ratio $E^{\prime} / E$, which, of course, according to Galois theory, does not possess any general solution in terms of radicals. With diligence, a particular solution may be found, but instead we shall discuss approximate solutions in the section after next, where we investigate into the experimental verifiability of the Doppler shifts (105). But first we must attend to a theoretical and conceptual issue of utmost significance.

\section{HOW FAST DOES TIME FLOW?}

It is evident that the conception of time afforded by the present theory, as encapsulated within the fundamental line element (30), is profoundly unorthodox. In particular, in addition to motion, time now depends also on the phase space evolution of systems that record it, and hence would be different, in general, for different recording systems. Moreover, this new conception of time dispels, at a stroke, the spell of the 'block' view of time [20], which is widely thought to be an inevitable byproduct of Einstein's special relativity. According to this 'block' view, since in the Minkowski picture time is as 'laid out' a priori as space, and since space clearly does not seem to 'flow', what we perceive as a 'flow of time', or 'becoming', must be an illusion. Worse still, in Einstein's theory, the relativity of simultaneous events demands that what is 'now' for one inertial observer cannot be the same, in general, for another. Therefore, to accommodate 'nows' of all possible observers, events must exist a priori, all at once, across the whole span of time [36]. As Weyl once so aptly put it, "The objective world simply is, it does not happen" 37]. Einstein himself was quite painfully aware of this shortcoming of his theories of relativity - namely, of their inability to capture the continual slipping away of the present moment into the unchanging past 38]. To be sure, the alleged unreality of this transience of 'now', as asserted by the 'block' view of time, is far from being universally accepted (see, e.g., [2, 39, 40]). However, what remains unquestionable is the fact that there is no explicit assimilation of such a transience in any of the established theories of fundamental physics.

By contrast, in the present theory, where proper time is defined by (30), the 'block' view of time endorsed by Weyl cannot be sustained. For time is now as much a 'state dependent' attribute of the world as states are time dependent attributes, and as the states of the world do 'happen' and 'become', so does time. To appreciate this dynamic nature of proper time as defined by (30), let us return 
once again to our clock that is moving and evolvingnow possibly non-uniformly - from, say, an event-state $\left(\mathrm{e}_{1}, \mathrm{~s}_{1}\right)$ to an event-state $\left(\mathrm{e}_{5}, \mathrm{~s}_{5}\right)$, in the combined space $(\mathcal{E}, \xi)$ (see FIG. 1). According to the line element (30), the proper duration recorded by the clock would be

$$
\Delta \tau=\int_{\left(\mathrm{e}_{1}, \mathrm{~s}_{1}\right)}^{\left(\mathrm{e}_{5}, \mathrm{~s}_{5}\right)} \frac{1}{\gamma(\theta)} d t
$$

where $\gamma(\theta)$ is defined by (26) as before. Now, assuming for simplicity that the clock is not massless, we can represent its journey by the integral curve of a timelike vector field $V^{A}$ on $(\mathcal{E}, \xi)$, defined, naturally, by

$$
V^{A}:=l_{P} \frac{d z^{A}}{d \tau} \stackrel{*}{=} \gamma(\theta)\left(c, l_{P} \theta^{I}\right)
$$

$(I=1,2,3, \ldots, 3+2 n)$, such that its external components $V^{a}(a=0,1,2,3)$ would trace out, for each possible state $s_{i}$ of the clock, the familiar four-dimensional timelike world-lines in the Minkowski spacetime $(\mathcal{M}, \eta)$. In other words, $V^{A}$ would give rise to the usual timelike, future-directed, never vanishing, 4-velocity vector field $V^{a}$ for the clock, tangent to each of the external timelike world-lines. As a result, the 'length' of the overall enveloping world-line would be given by the proper time (107), whereas the 'length' of the external world-line, for a given $s_{i}$, would be given by the Einsteinian proper time

$$
\Delta t_{E}^{i}=\int_{e_{1}}^{e_{i}} \frac{1}{\gamma(v)} d t
$$

with $\gamma(v)$ being the usual Lorentz factor given by (7). In FIG. 1, five of such external timelike world-linesone for each $s_{i}(\mathrm{i}=1,2,3,4,5)$ - are depicted by the blue curves with arrowheads going 'upwards', and the overall enveloping world-line traced out by $V^{A}$ is depicted by the dashed green curve going from the event-state $\left(e_{1}, s_{1}\right)$ to the event-state $\left(e_{5}, s_{5}\right)$. It is at once clear from this picture that the external world-line of the clock is not given all at once, stretched out till eternity, but 'grows' progressively further as time passes, with each temporally successive stage of the evolution of the clock, like a tendril on a garden wall. In fact, from FIG. 1, line elements (22) and (3), and the condition (18), it is easy to see that the instantaneous directional rate of this growth is simply

$$
U^{a}=\frac{V^{a}}{\sqrt{-\eta_{b c} V^{b} V^{c}}} \frac{d t_{E}}{d y}, \quad \text { with } \quad \frac{d t_{E}}{d \mathrm{y}} \geq t_{P},
$$

where $d y:=|d \mathbf{y}|$ is the infinitesimal dimensionless phase space distance between the two successive states of the clock (cf. definition (12)), and $d t_{E}$ is the usual infinitesimal Einsteinian proper duration defined by (3). Thus 'now' for the clock (depicted by the red dot in the FIG. 1) moves in the future direction along its world-line, at the rate of no less than one Planck unit of time per Planck unit of change in its physical state. Crucially, since the 4velocity of an observer can never vanish, the lower bound

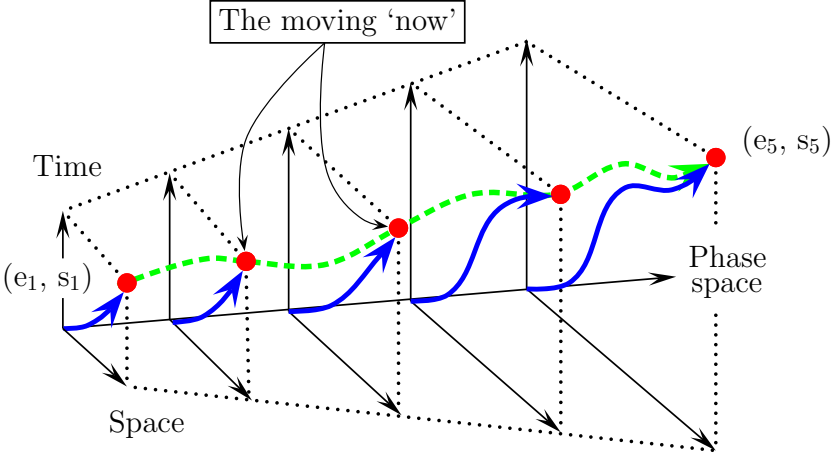

FIG. 1: Space-time-state diagram depicting the 'flow of time'. (A similar diagram was used pejoratively in 22] to describe and discredit Dunne's early attempt [41, 42] to capture the 'flow of time' in physics. The objections raised in 22$]$ and 43] against Dunne's failed attempt are not applicable here.)

on the above rate shows that not only the 'now' moves, but it cannot not move. To parody Weyl quoted above, the objective world cannot simply be, it can only happen.

To consolidate this conclusion, let us note that even the overall enveloping world-line (the dashed green curve in FIG. 1) cannot remain 'static' in the present scenario. This can be seen by first parallelling the above analysis for the $(1+2 n)$-dimensional 'internal' space $(\mathcal{O}, \zeta)$, instead of the external spacetime $(\mathcal{M}, \eta)$. In the FIG. 1 this amounts to slicing up the combined space $(\mathcal{E}, \xi)$ along the phase space axis instead of the spatial axis, and then observing that even the 'internal' world-line cannot but 'grow' progressively further as time passes, at the rate of

$$
U^{\alpha}=\frac{l_{P} V^{\alpha}}{\sqrt{-\zeta_{\beta \gamma} V^{\beta} V^{\gamma}}} \frac{d t_{H}}{d \mathrm{x}}, \quad \text { with } \quad \frac{d t_{H}}{d \mathrm{x}} \geq c^{-1}
$$

where $V^{\alpha}$ is the 'internal' part of the vector field $V^{A}$, $d \mathrm{x}:=|d \mathbf{x}|$ is the infinitesimal spatial distance between two slices, and $d t_{H}$ is the infinitesimal 'internal' proper duration defined by (17). Thus, the 'now' for the clock moves in the future direction, along its 'internal' worldline, also in the 'internal' space $(\mathcal{O}, \zeta)$. Consequently, even the overall world-line - namely, the dashed green curve in the FIG. 1-cannot be 'static', but 'grows' at the combined, instantaneous, directional rate of

$$
U^{A}=\left(\frac{V^{a}}{\sqrt{-\eta_{b c} V^{b} V^{c}}} \frac{d t_{E}}{d \mathrm{y}}, \frac{l_{P} \omega^{\mu}}{\sqrt{-\zeta_{\beta \gamma} V^{\beta} V^{\gamma}}} \frac{d t_{H}}{d \mathrm{x}}\right),
$$

where $\omega^{\mu} \equiv V^{\mu}$ is the instantaneous evolution rate for the clock. What is more, this overall rate of motion for the 'now' also cannot vanish. This can be seen easily by using the rest frame for the clock to evaluate the magnitude of $U^{A}$, and then using the lower bound from (110) to obtain the lower bound on this magnitude, which yields

$$
\sqrt{-\xi_{A B} U^{A} U^{B}} \geq t_{P} .
$$


Thus, in the present theory, not only does the external 'now' move along timelike world-lines, but there does not remain even an overall 'block' - such as a 'static' space $(\mathcal{E}, \xi)$ - that could be used to support a 'block' view of time. That is to say, the new conception of 'becoming' embedded in the structure $(\mathcal{E}, \xi)$ is truly Heraclitean [20].

It is also worth noting that, in the present theory, even the four-dimensional spacetime continuum no longer enjoys the absolute status it does in Einstein's theories of relativity. Einstein dislodged the older concepts of 'absolute time' and 'absolute space', only to be replaced by the new framework of 'absolute spacetime' - namely, a continuum of in principle observable events, idealized as a connected pseudo-Riemannian manifold with observerindependent spacetime intervals. Since it is impossible to directly observe this remaining absolute structure without recourse to the behaviour of material objects, it is perhaps best viewed as the 'ether' of the modern times [4]. By contrast, it is evident from both the fundamental quadratic invariant (30) and the FIG. 1 above, that in the present theory this four-dimensional spacetime continuum no longer has the absolute, observer-independent meaning. In fact, apart from the laws of nature, there is very little absolute structure left in the present theory, for even the quadratic invariant (30) is dependent on the phase space structure of the material system being observed. That is to say, even the manifold $(\mathcal{E}, \xi)$ that replaces the Minkowski spacetime $(\mathcal{M}, \eta)$ in the present theory does not have the absolute status, as its construction is not independent of the system being observed.

The presence of the absolute spacetime continuum in Einstein's theories of relativity makes them conducive to the 'block' view of time, as we discussed above. In particular, they are generally regarded as compatible with the tense-less notions of time. To be sure, along the timelike world-lines of material objects, the times of events in these theories are linearly ordered relative to each other by the transitive, asymmetric, and irreflexive relation 'precedes' (see, e.g., [39]). But there is no explicit reference to absolute past, present, or future in this purported relation. And, in essence, it is this lack of any reference to such tenses in the relation 'precedes' along the worldlines that is responsible for the recurring speculations on 'time travel' based on these theories (see, e.g., 45]). For, if time does not 'flow' from the past to the future via the present, then it is no different in nature from space, and hence the instants of time should be as traversable as the places in space are. By contrast, in the theory developed here time does 'flow' from the determined past to the undetermined future, and hence 'time travel' is in principle inconceivable. Consequently, over and above its intrinsic necessity, an experimental verification of the predictions of the present theory acquires an added incentive. And to that empirical possibility we turn next.

\section{EXPERIMENTAL VERIFIABILITY}

It is worth noting that, although unity in the Planck units of the quantum gravity regime, in the m.k.s. units of everyday physics the factor $t_{P}$ of the present theory that converts state space distances into temporal units is some 35 orders of magnitude smaller than the factor $c^{-1}$ of special relativity that converts spatial distances into temporal units. Even in the 'natural units' of the regime of particle (or quantum) physics where $\hbar=c=1$, the former conversion factor is some 28 orders of magnitude smaller than the latter. And, of course, it is this minuteness of the Planck time that is responsible for the lack of positive experimental data on physics at that scale.

In recent years, however, there have been a number of attempts to remedy this dire state of affairs (see, e.g., 15, 16, 17, 18, 19 and references therein). Most of these attempts revolve around Planck scale modifications of the usual special relativistic dispersion relation - analogous to the one considered above (cf. [69) ). The modifying terms in these relations are usually either linearly or quadratically suppressed by the Planck energy, and one relies on some astrophysical phenomena - such as the gamma-ray bursts - to obtain observational bounds on the deviations from the special relativistic predictions. As long as these deviations are linearly suppressed by the Planck energy, astrophysical observations do tend to put useful bounds on them. However, when the deviations happen to be quadratically suppressed by the Planck energy - as is the case in the relation (69) proposed above - then the minuteness of the deviations renders even such high energy astrophysical strategy for their detection far less promising. And yet, it has been argued in Ref. 17] that advanced cosmic-ray observatories and neutrino observatories that have been planned to be operational in the near future may provide experimental possibilities to test such quadratically suppressed deviations (cf. also [19]). If this optimism of Ref. [17] turns out to be justified, then the dispersion relations (69) predicted by the present theory may also be subjected to a test by the same means. Since this and related possibilities are already extensively discussed in the literature cited above, we shall not dwell on them any further. Instead, we shall concentrate on a different possibilitythat of a verifiability of the Doppler shift formula (105) predicted by the present theory.

As mentioned towards the end of Sec. VI, it is rather difficult to solve the relation (105) exactly, but it can be approximated - even at high energies - to yield a practical result. Actually, since the experimenter is usually a receiver rather than a source of the radiation, we shall use an equivalent formula for Doppler shifts, namely

$$
\frac{E^{\prime}}{E}=\frac{\sqrt{\left(\varepsilon^{\prime}\right)^{2}-\frac{v^{2}}{c^{2}}}}{\varepsilon^{\prime}\left[\varepsilon^{\prime}+\frac{v}{c} \cos \phi^{\prime}\right]},
$$

written in terms of the angle $\phi^{\prime}$-as determined by the receiver-between the relative velocity and photon mo- 
mentum. This angle is related to the corresponding angle $\phi$ determined by the source via the following generalized and energy-dependent aberration relation

$$
\cos \phi^{\prime}=\frac{\varepsilon^{\prime} \cos \phi-\frac{v}{c}\left(\varepsilon^{\prime}\right)^{-2}+\frac{c}{v}\left(1-\left(\varepsilon^{\prime}\right)^{2}\right)}{\varepsilon^{\prime}-\frac{v}{c} \cos \phi},
$$

which follows from the expression (102) and a pair of relations such as (104). In the rigorous limit $E / E_{P} \rightarrow 0$ this aberration relation reduces to the usual one derived by Einstein almost a hundred years ago. It can be used, for instance, to obtain (114) from (105). Of course, (114) can also be easily derived directly from (101), just as we derived (105) from (99) in the section before the last.

Now, a Maclaurin expansion of the right hand side of (114) around the value $E / E_{P}=0$, after keeping terms only up to the second order in the ratio $E / E_{P}$, gives

$$
\begin{aligned}
\frac{E^{\prime}}{E} \approx & \frac{\sqrt{1-\frac{v^{2}}{c^{2}}}}{1+\frac{v}{c} \cos \phi^{\prime}} \\
& +\frac{1}{2} \frac{E^{2}}{E_{P}^{2}}\left[\frac{1-2 \frac{v^{2}}{c^{2}}-\frac{v^{3}}{c^{3}} \cos \phi^{\prime}}{\left(1+\frac{v}{c} \cos \phi^{\prime}\right)^{2} \sqrt{1-\frac{v^{2}}{c^{2}}}}\right]\left(1-\frac{E^{\prime}}{E}\right)^{2}+\ldots
\end{aligned}
$$

This truncation is an excellent approximation to (114). Even for TeV photons, the next term in the expansion$E^{4} / E_{P}^{4}$-is of the order of $10^{-64}$. The quadratic equation (116) can now be solved for the desired ratio $E^{\prime} / E$, and then the physical root once again expanded, now in the powers of $v / c$. In what results if we again keep terms only up to the second order in the ratio $E / E_{P}$, then, after some straightforward algebra, we arrive at

$$
\frac{E^{\prime}}{E} \approx 1-\frac{v}{c} \cos \phi^{\prime}+\left[\left(1+\frac{1}{2} \frac{E^{2}}{E_{P}^{2}}\right) \cos ^{2} \phi^{\prime}-\frac{1}{2}\right] \frac{v^{2}}{c^{2}}+\ldots
$$

In the limit $E \ll E_{P}$ this expansion clearly reduces to

$$
\frac{E^{\prime}}{E} \approx 1-\frac{v}{c} \cos \phi^{\prime}+\left[\cos ^{2} \phi^{\prime}-\frac{1}{2}\right] \frac{v^{2}}{c^{2}}+\ldots,
$$

which is simply the familiar special relativistic result.

Comparing (117) and (118) we see that up to the first order in $v / c$ there is no difference between the special relativistic result and that of the present theory. The first deviation between the two theories occur in the secondorder coefficient, precisely where special relativity differs also from the classical theory. What is more, this secondorder deviation depends non-trivially on the angle between the relative velocity and photon momentum. For instance, up to the second order, both red-shifts $\left(\phi^{\prime}=0\right)$ and blue-shifts $\left(\phi^{\prime}=\pi\right)$ predicted by (117) differ significantly from those predicted by special relativity. In particular, the red-shifts are now somewhat less red-shifted, whereas the blue-shifts are somewhat more blue-shifted. On the other hand, the transverse red-shifts $\left(\phi^{\prime}=\pi / 2\right.$

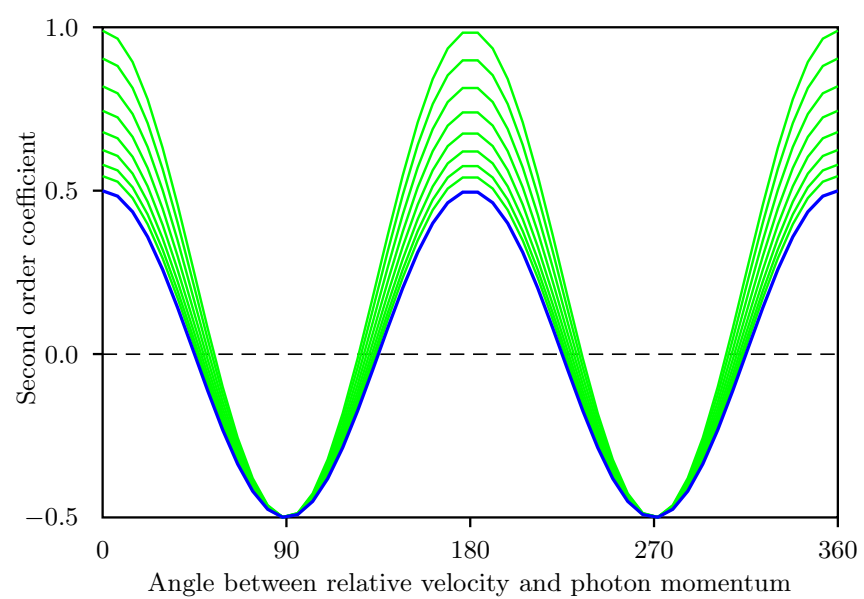

FIG. 2: The energy-dependent signatures of refined relativity. The green curves are based on the predictions of the present theory, for $E / E_{P}=0.3$ to 0.99 in the ascending order, whereas the blue curve is based on the prediction of special relativity.

or $3 \pi / 2$ ) remain identical to those predicted by special relativity. As a result, even for the photon with energy approaching the Planck energy, an Ives-Stilwell type classic experiment 46] would not be able to distinguish the predictions of the present theory from those of special relativity. The complete angular distribution of the secondorder coefficient predicted by the two theories, along with its energy dependence, is displayed in the FIG. 2.

In spite of this non-trivial angular dependence of Doppler shifts, in practice, due to the quadratic Planck energy suppression, distinguishing the expansion (117) from its special relativistic counterpart (118) would be a highly non-trivial task. This can also be inferred from FIG. 2, which makes it clearer that a near-future verification of the prediction (117) by means of a terrestrial experiment is highly unlikely. The maximum laboratory energy that may be available to us is of the order of $\mathrm{TeV}$, yielding $E^{2} / E_{P}^{2} \sim 10^{-32}$. This represents a correction of one part in $10^{32}$ from (118), demanding a phenomenal sensitivity for its detection, well beyond the means of the state-of-the-art precision technology.

Let us see if we can do better than this by using an extraterrestrial source. For example, suppose we attempt to distinguish between the second order Doppler shifts predicted by the two theories using a binary pulsar emitting $\gamma$-ray pulses. Of course, most of the known pulsars are radio sources, but a handful of them are indeed $\gamma$ ray pulsars emitting photons of energy in the range of $\mathrm{TeV}$ (see, e.g., 47]). Now, it is well known that binary pulsars not only exhibit Doppler shifts, but the secondorder shifts resulting from the rapid motion of a pulsar orbiting about its companion can be isolated, say, from the first order shifts, because they depend on the square of the relative velocity, which varies as the pulsar moves along its two-body elliptical orbit (see, e.g., [48]). Due to these Doppler shifts, the rate at which its pulses are observed on Earth reduces slightly when the pulsar is re- 
ceding from the Earth in its orbit $\left(\phi^{\prime}=0\right)$, compared to when it is approaching the Earth $\left(\phi^{\prime}=\pi\right)$. The parameter relevant in the 'time of arrival' analysis of these pulses is variously called the 'Red-shift-Doppler parameter' or the 'time dilation parameter' [4], which is a non-trivial function of the gravitational red-shift, the masses of the two binary stars, and other Keplerian parameters. For a radio pulsar also exhibiting periastron precession similar to the perihelion advance of Mercury, this parameter can be determined with an excellent precision. This is, of course, the case for the most famous pulsar: PSR $1913+16$ 49]. Now, the arrival times of the pulses of this pulsar - which have been monitored for three decadesare extremely sensitive to the 'time dilation' parameter, and thereby to the second-order Doppler shifts brought about by its orbital motion. What is more, the overall precision on the timing of the pulses of PSR 1913+16, and consequently that of its periastron advance, is famously better than one part in $10^{12}$. Indeed, the monitoring of the decaying orbit of PSR $1913+16$ constitutes one of the most precise tests of general relativity to date.

Encouraged by these facts, one might hope that - at least in principle - similar careful observations of a suitable $\gamma$-ray pulsar may be able to distinguish the predictions of the present theory from those of special relativity. However, the highest energy radiating pulsar known to date emits $\gamma$-ray photons of energy no greater than 10 $\mathrm{TeV}$, giving the discriminating ratio $E^{2} / E_{P}^{2}$ to be of the order of $10^{-30}$, which is only two orders of magnitude improvement from a possible terrestrial scenario. Thus, it appears that, even with such an exotic astrophysical source as a $\gamma$-ray pulsar, it would be quite a challenge to distinguish between the predictions of the two theories.

Facing this difficulty, one may ask a converse question: How energetic the $\gamma$-rays emitted by a pulsar have to be to meet the achievable precision? Even if we dare to go by the remarkable precision available on PSR $1913+16$, the answer would have to be: exceeding $10^{10} \mathrm{TeV}$.

Of course, no such ultra-high energy pulsar has been found. Worse still, there are reasons to believe - at least within the standard framework of special relativity - that none above $10 \mathrm{TeV}$ can ever be found. Above the 10 $\mathrm{TeV}$ threshold, $\gamma$-rays are expected to severely attenuate through pair-production in the intervening infrared background long before reaching the Earth. There are, however, good indications that this theoretical threshold is, in fact, not respected by nature [50, 51]. Perhaps within a refined inertial structure, such as the one being proposed here, there is no such threshold (cf. [16, 18]). Whatever turns out to be the resolution of this conflict, there may be independent reasons for a pulsar to refrain from emitting radiations of energy as high as $10^{10} \mathrm{TeV}$. For instance, the intrinsic dynamics of the magnetically trapped charged particles responsible for producing the radiation emitted by the pulsars may be subject to its very own high energy threshold. We simply do not know. On balance, however, we cannot rule out the possibility of finding in the future a suitable $\gamma$-ray binary pulsar emitting radiation of energies exceeding $10^{10} \mathrm{TeV}$.

The discussion above is clearly meant to be indicative of the difficulties rather than that of realistic possibilities. And even then we face almost insurmountable obstacles to our aim. This is a great pity, not the least because an experimental test of the present theory has a direct bearing on one of the oldest disputes in natural philosophy: Does the perceived 'flow of time' reflect a genuinely structural attribute of the world, as the present theory maintains, or is it an illusion, as special relativity seems to suggest? In view of the issues discussed in the previous section, an empirical corroboration of a signature predicted by the present theory would lend much needed support to the former-Heraclitean-notion of time.

\section{CONCLUDING REMARKS}

Perhaps one of the most attractive features of the above theory is its economy of thought, parallelling the rationale of Einstein's special relativity. In particular, unlike the two-scales theories mentioned in the Introduction, the present theory is based on only one observerindependent fundamental scale, namely, the inverse of the Planck time, and this fact makes it a truly Planck scale rooted theory. To be sure, the vacuum speed of light also remains an invariant in the theory, but it plays only a secondary and derivative role. And yet, this generalization of Einstein's second postulate does not necessitate any compromise with his first postulate, namely, the principle of relativity. Quite the contrary, a more complete implementation of this basic principle offers at least four-fold improvement over his special relativity. First, it allows one to eliminate the dualistic notion of time pervasive in our physical theories - from classical to quantal - that has caused so many conceptual difficulties in our understanding of 'quantum gravity'. Second, it allows one to capture and quantify the elusive 'flow of time' as a genuinely structural attribute of the world. Third, it renders redundant one of the last remnants of 'ether' - namely, the absolute spacetime-from the local inertial structure. And fourth, it allows one to eliminate the unphysical concepts of unbounded energies, momenta, lengths, and durations from a physical theory.

These are more than sufficient reasons to take the present theory seriously, despite its unorthodox appeal. But the theory has even more to offer. For example, it provides a natural contraception for the speculations on time travel. A prerequisite for the possibility of time travel is a tenseless structure of spacetime, necessitated by the special and general theories of relativity. By contrast, the notions of past, present, and future are intrinsic to the spacetime structure proposed in the present theory, and hence time travel becomes in principle inconceivable.

Another somewhat related implication of the present theory concerns the thermodynamic arrow of time. It is well known that - from Boltzmann to Prigogine - no one has succeeded in explaining the thermodynamic unidi- 
rectionality of time in terms of micro-physics, essentially because the microscopic laws of physics happen to be symmetric in time. But according to the present theory the local causal structure intrinsically distinguishes the future from the past, due to the built-in directional 'flow of time', and thereby provides an opportunity to derive the thermodynamic arrow of time from the timesymmetric microscopic laws. This aspect of the proposed theory has not been discussed in the present paper, but it will be explicated fully in a separate publication.

In addition to these theoretical implications, several phenomenological aspects of the present theory are also worth noting. As mentioned before, several attempts to construct a theory of quantum gravity predict energydependent deviations from the dispersion relations based on special relativity. The present theory shows that such deviations can be understood on the basis of the first principles of a theory, thereby providing a natural physical understanding of the deviations. The prediction of these deviations, along with the deviations from the special relativistic Doppler shifts, suggest that the present theory may be viewed also as a non-artificial test-theory for the experimental investigations of special relativity.

Given the refined local inertial structure embedded in the quadratic invariant (30), the next natural challenge, of course, is to understand how this new structure refines the classical - and, indeed, the quantumconceptions of gravity as prescribed by the principle of equivalence. This challenge, with quite a broad understanding of the term 'quantum gravity', will be taken up and addressed fully in a companion paper.

\section{Acknowledgments}

I am deeply indebted to my mentor Abner Shimony for his kind, generous, and sustained financial support without which this work would not have been possible.
[1] A. Einstein, Preussische Akademie der Wissenschaften (Berlin). Sitzungsberichte: 688 (1916).

[2] J. Christian, in Physics Meets Philosophy at the Planck Scale, edited by C. Callender and N. Huggett (Cambridge University Press, Cambridge, UK, 2001), pp. 305-338.

[3] R.M. Wald, General Relativity (University of Chicago Press, Chicago, 1984), pp. 4-6.

[4] W. Heisenberg, Z. Physik 110, 251 (1938).

[5] B. Carazza and H. Kragh, Am. J. Phys. 63, 595 (1995).

[6] H.S. Snyder, Phys. Rev. 71, 38 (1947).

[7] C.N. Yang, Phys. Rev. 72, 874 (1947).

[8] G. Amelino-Camelia, Phys. Lett. B 510, 255 (2001).

[9] J. Magueijo and L. Smolin, Phys. Rev. Lett. 88, 190403 (2002).

[10] J. Kowalski-Glikman, Phys. Lett. B 547, 291 (2002).

[11] J. Lukierski and A. Nowicki, Int. J. Mod. Phys. A 18, 7 (2003).

[12] D.V. Ahluwalia-Khalilova, arXiv:gr-qc/0212128

[13] G. Ellis, Nature 422, 563 (2003).

[14] V.A. Kostelecký and S. Samuel, Phys. Rev. D 39, 683 (1989).

[15] L. Smolin, arXiv:hep-th/0303185

[16] J. Alfaro and G. Palma, Phys. Rev. D 67, 083003 (2003).

[17] G. Amelino-Camelia, Int. J. Mod. Phys. D 12, 1633 (2003).

[18] S. Sarkar, Mod. Phys. Lett. A 17, 1025 (2002).

[19] T. Jacobson, S. Liberati, and D. Mattingly, Phys. Rev. D 67, 124011 (2003).

[20] K. Popper, The World of Parmenides: Essays on the Pre-Socratic Enlightenment (Routledge, London, 1998).

[21] P.C.W. Davies, The Physics of Time Asymmetry (Surrey University Press, 450 Edgware Road, London, 1974).

[22] M. Lockwood, Mind, Brain and the Quantum (Blackwell Publishers, Oxford, 1989), p. 264.

[23] H. Salecker and E.P. Wigner, Phys. Rev. 109, 571 (1958).

[24] V.I. Arnold, Mathematical Methods of Classical Mechanics, 2nd edition (Springer-Verlag, New York, 1989).

[25] R. Penrose, Gen. Relativ. Gravit. 28, 581 (1996).

[26] C.J. Isham, in Integrable Systems, Quantum Groups, and
Quantum Field Theories, edited by L.A. Ibort and M.A. Rodriguez (Kluwer, Dordrecht, 1993), pp. 157-288.

[27] H. Goldstein, Classical Mechanics, 2nd edition (AddisonWesley, Reading, 1980), pp. 570-575.

[28] V. Ambarzumian and D. Iwanenko, Z. Phys. 64, 563 (1930).

[29] Y. Choquet-Bruhat, C. DeWitt-Morette, and M. DillardBleick, Analysis, Manifolds and Physics, Revised edition (Elsevier Science Publishers B.V., North-Holland, 1982).

[30] J. Anandan, Phys. Lett. A 147, 3 (1990).

[31] D.I. Fivel, Phys. Rev. A 50, 2108 (1994).

[32] J.R. Klauder, Foundations of Physics 27, 1467 (1997).

[33] J.R. Klauder, arXiv:quant-ph/0112010

[34] E.F. Taylor and J.A. Wheeler, Spacetime Physics, 2nd edition (W.H. Freeman and Company., New York, 1992).

[35] S. Judes and M. Visser, Phys. Rev. D 68, 045001 (2003).

[36] R. Penrose, The Emperor's New Mind (Oxford University Press, Oxford, 1989), pp. 303.

[37] H. Weyl, Philosophy of Mathematics and Natural Science (Princeton University Press, Princeton, 1949), p. 116.

[38] R. Carnap, in The Philosophy of Rudolf Carnap, P.A. Schilpp (ed.) (LaSalle, IL: Open Court, 1963), pp. 37-38.

[39] A. Shimony, Search for a Naturalistic World View, Vol. II (Cambridge University Press, Cambridge, 1993), p. 271.

[40] A. Shimony, in The Geometric Universe: Science, Geometry, and the Work of Roger Penrose, S.A. Huggett et al. (eds) (Oxford University Press, Oxford, 1998), p. 161.

[41] J.W. Dunne, An Experiment with Time (A. and C. Black, London, 1927).

[42] J.W. Dunne, The Serial Universe (Faber and Faber Ltd., London, 1934).

[43] C.D. Broad, Philosophy 10, 168 (1935).

[44] A. Einstein, in The Collected Papers of Albert Einstein, Vol. 7, translated by Alfred Engel (Princeton University Press, Princeton, 2002), pp. 160.

[45] S. Krasnikov, Phys. Rev. D 65, 064013 (2002).

[46] H.E. Ives and G.R. Stilwell, J. Opt. Soc. Am. 28, 215 (1938).

[47] A.M. Atoyan et al., Astron. Astrophys. 383, 864 (2002). 
[48] C.M. Will, Theory and Experiment in Gravitational Physics, revised edition (Cambridge University Press, Cambridge, 1993), chapter 12.

[49] C.M. Will, Living Rev. Rel. 4, 4 (2001); arXiv:gr-qc/0103036
[50] F. Aharonian, et al., Astron. Astrophys. 349, 11 (1999).

[51] D. Finkbeiner, M. Davis, and D. Schlegel, Astrophys. J. 544, 81 (2000). 